\title{
Variabilité du débourrement végétatif du sapin de Céphalonie (Abis cephalonica Loudon) en plantation
}

\author{
B Fady 1
}

avec la collaboration technique de D Vauthier 2

\begin{abstract}
1 Université Aix-Marseille 3, faculté des sciences de St-Jérôme, laboratoire de botanique et écologie méditerranéenne, avenue Escadrille Normandie-Niemen, 13997 Marseille cedex 13;

2 INRA, station expérimentale du Ruscas, RN 98, 83230 Bormes-les-Mimosas, France
\end{abstract}

(Reçu le 24 février 1990; accepté le 10 octobre 1990)

\begin{abstract}
Résumé - L'étude phénologique du débourrement végétatif du sapin de Céphalonie a été réalisée sur le bourgeon terminal de la tige principale d'individus âgés de 11 ans. L'échantillon, représenté sur la plantation comparative de Saint-Lambert (Vaucluse), était composé de 9 provenances, dont quatre étaient subdivisées en descendances maternelles. L'utilisation d'une méthode d'observation qualitative en 5 points (Debazac, 1965 et 1967) apporte une précision supplémentaire significative dans la discrimination des provenances par rapport à une méthode indiquant seulement l'état débourré ou non débourré du bourgeon. La précision des 2 méthodes d'observation est relativement comparable pour le calcul des paramètres génétiques. La variabilité interprovenance du débourrement s'est révélée significative : la population insulaire de Céphalonie et surtout les populations orientales de l'aire de répartition naturelle (Eubée et Parnis) sont plus précoces que celles du Péloponnèse et de la Grèce continentale. La précocité ou la tardivité du débourrement des populations a été envisagé sous la forme d'une stratégie adaptative en fonction de l'importance relative des gelées tardives et du stress hydrique estival. Cette précocité est par ailleurs nettement corrélée avec une faible croissance en hauteur. L'héritabilité de la phénologie du débourrement est plus faible qu'attendue, sans doute du fait d'un effet environnement notable pendant l'année d'observation. Elle est cependant suffisante pour permettre d'envisager une sélection de provenances et descendances tardives et vigoureuses, susceptibles d'être installées en reboisement en France méditerranéenne.
\end{abstract}

débourrement végétatif / variabilité génétique / adaptation / héritabilité / Abies cephalonica

Summary - Variation of vegetative flushing in Abies cephalonica Loudon in an experimental site. The study of vegetative flushing in Abies cephalonica was performed on the main shoot terminal bud of 11-year-old trees. The tree collection was grown at the Saint-Lambert experimental site (Vaucluse, Southern France) and consisted of 9 provenances, 4 of which were divided into progenies. Precision was significantly increased using a 5-point observation method (Debazac, 1965, 1967) compared to a 2-point flushed/non-flushed method for between-provenance data, but showed only a slight increase among provenance genetic parameters. Bud flushing variability proved to be significant between provenances: the island population of Cephalonia and the populations from the eastern part of the natural distribution area (Euboea and Parnis) flush earlier than Peloponnesus 
and continental Greece populations. The development of early or late flushing adaptive strategies in relation to the occurrence of either early summer water stress or late spring frost is discussed. Early flushing was significantly correlated with slow height growth. Heritability of vegetative flushing was lower than expected, probably due to high environmental interaction during the experiment. However, heritability had a sufficiently high value to make selection of late flushing and fast growing provenances and progenies possible in use for reforestation in Mediterranean France.

vegatative flushing / genetic variability / adaptation / heritability / Abies cephalonica

\section{INTRODUCTION}

Le débourrement est un caractère phénologique très important dans l'étude de la stratégie adaptative des populations forestières et dans le choix des semenciers pour leur régénération. Les individus les plus précoces sont sensibles aux gelées printanières et, en région méditerranéenne, les plus tardifs sont pénalisés visà-vis du stress hydrique estival. Le sapin de Céphalonie est une espèce très précoce par rapport aux autres sapins de type méditerranéen (Fady et al, 1990) et la mise en évidence de provenances et d'individus relativement tardifs n'en est que plus importante pour son utilisation en reboisement en France méditerranéenne.

Par ailleurs, ce caractère phénologique et sa variation permettent généralement une bonne différentiation des provenances d'une espèce forestière (Kozlowski, 1964; Lacaze et Tomassone, 1967 pour Abies grandis; Lacaze, 1969 pour Picea abies; Arbez, 1969 pour Abies alba; Birot et Ferrandès, 1972 et White et al, 1979 pour Pseudotsuga menziesii; Steiner, 1979 pour le genre Pinus; Lavender, 1980; Worrall, 1983 pour Abies amabilis et $A$ lasiocarpa). Les massifs forestiers qui abritent les peuplements de sapin de Céphalonie étant largement dispersés et caractérisés par des conditions écologiques variées (Barbero et Quézel, 1976; Fady, 1990), il est possible de s'attendre à l'expression de réponses phénologiques variées.
Dans l'étude qui suit, l'amplitude de la variabilité du caractère précocité du débourrement a été mesurée aux niveaux intra- et interprovenances. Une explication de l'allure de cette variabilité a été tentée en fonction des conditions bioclimatiques de l'aire d'origine.

\section{MATÉRIEL ET MÉTHODES}

\section{Matériel végétal}

Sur la plantation de Saint-Lambert (tableau I), 9 provenances de sapin de Céphalonie (tableau II et fig 1) ont été observées, chacune représentée par 100 individus repérés par un drapeau de couleur sur le terrain; les provenances Evia et Pnas ne présentaient que 69 et 74 individus observables respectivement. Afin de pouvoir calculer les paramètres génétiques, les provenances les mieux représentées au niveau familial ont été choisies. Quatre provenances seulement, situées sur la plantation VG1, avaient un nombre de familles supérieur à 10 (tableau III) : 3 originaires du Péloponnèse (Pnon, massif du Parnon; Erym, massif de l'Erymanthos; Veti, massif du Mainalon) et 1 de la côte orientale de la Grèce continentale (Evia, île d'Eubée).

\section{Méthode}

La phénologie du débourrement a été observée 7 fois consécutives à intervalles réguliers, du 28 avril au 19 mai 1987. Trois méthodes ont été utilisées pour observer le stade de débourrement 
Tableau I. Caractéristiques de la plantation de St-Lambert. Le dispositif a une structure générale en blocs incomplets et composition aléatoire. Les plants (2-2) ont été mis en terre au cours de l'hiver 1980-1981.

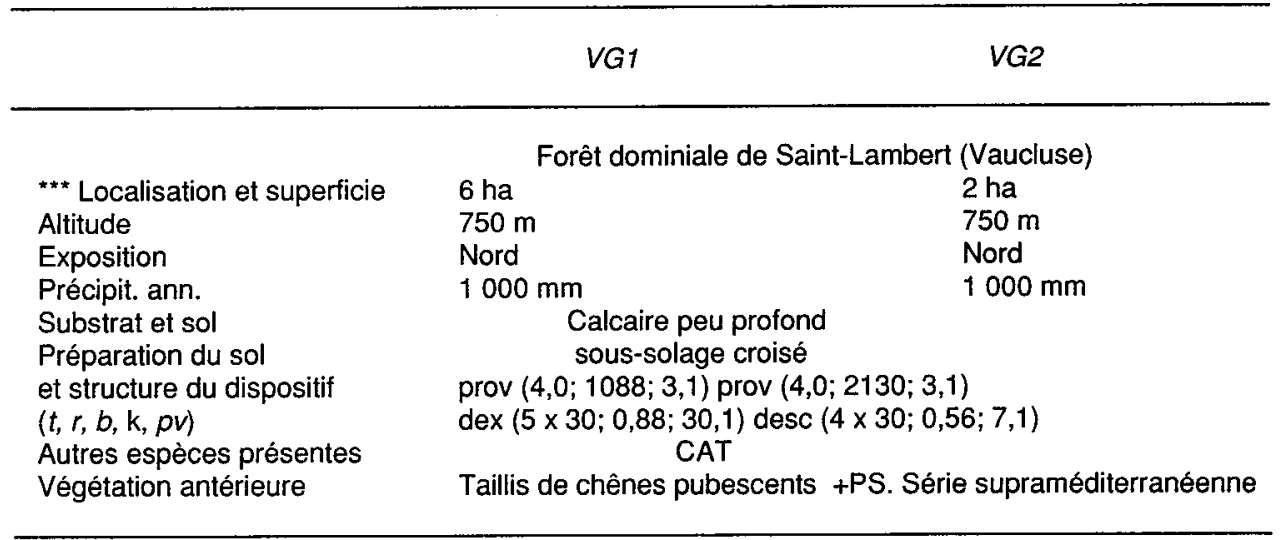

$t=$ nombre de traitements (provenances ou descendances); $r=$ nombre de répétitions; $b=$ nombre de blocs; $k=$ nombre de parcelles unitaires par bloc; $p u=$ nombre d'individus par parcelle unitaire; $C A T=$ Cedrus atlantica; $P S=$ Pinus sylvestris.

du bourgeon terminal situé à l'apex de l'axe principal.

\section{Méthode en 2 points (méthode 1-2)}

A chaque passage, le bourgeon est qualifié de 2 manières : débourré (note 2) ou non débourré (note 1).

\section{Méthode en 5 points (méthode 1-5, d'après Debazac, 1965 et 1967)}

Note 1 : bourgeon dormant;

note 2 : bourgeon gonflé, les écailles plus ou moins écartées;

note 3 : apparition des aiguilles par transparence sous la coiffe;

note 4 : bourgeon débourré, les aiguilles ont déchiré la coiffe;

note 5 : les aiguilles sont libérées de la coiffe et la pousse croît.

Ces 2 méthodes permettent de calculer une date de débourrement, notamment, pour une provenance, en choisissant l'événément « $50 \%$ d'individus débourrés" comme date moyenne de débourrement. En fait, pour étudier l'allure de la variation intraspécifique, un indice de précocité a été utilisé; il a été calculé en additionnant les 7 notes phénologiques successives. Cet indice est considéré comme un caractère quantitatif dans les traitements statistiques. Plus l'indice est élevé, plus la précocité du débourrement est grande.

\section{Méthode des sommes de températures}

Lorsque l'indice de débourrement de chaque individu est connu, il est remplacé par la somme des températures journalières supérieures à $0^{\circ} \mathrm{C}$ ou à $5^{\circ} \mathrm{C}$ entre le $1^{\text {er }}$ janvier de l'année et le jour du débourrement. Des intervalles de temps identiques entre observations sont ainsi remplacés par les sommes de températures correspondantes, a priori plus significatives sur le plan biologique (Mauget, 1983 et 1987), et qui permettent de prédire avec une bonne précision les dates de débourrement de plusieurs espèces de conifères (Thompson et Moncrieff, 


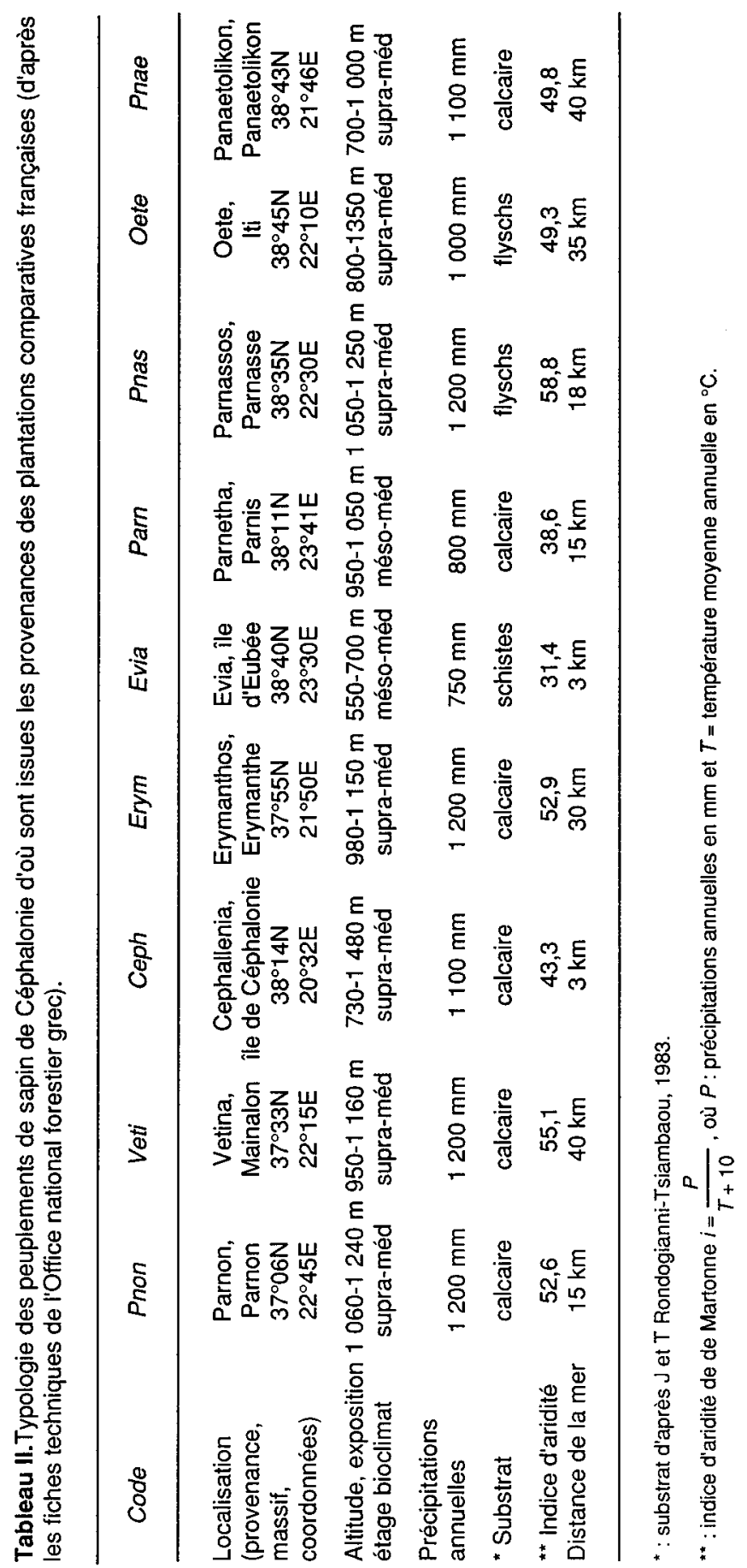




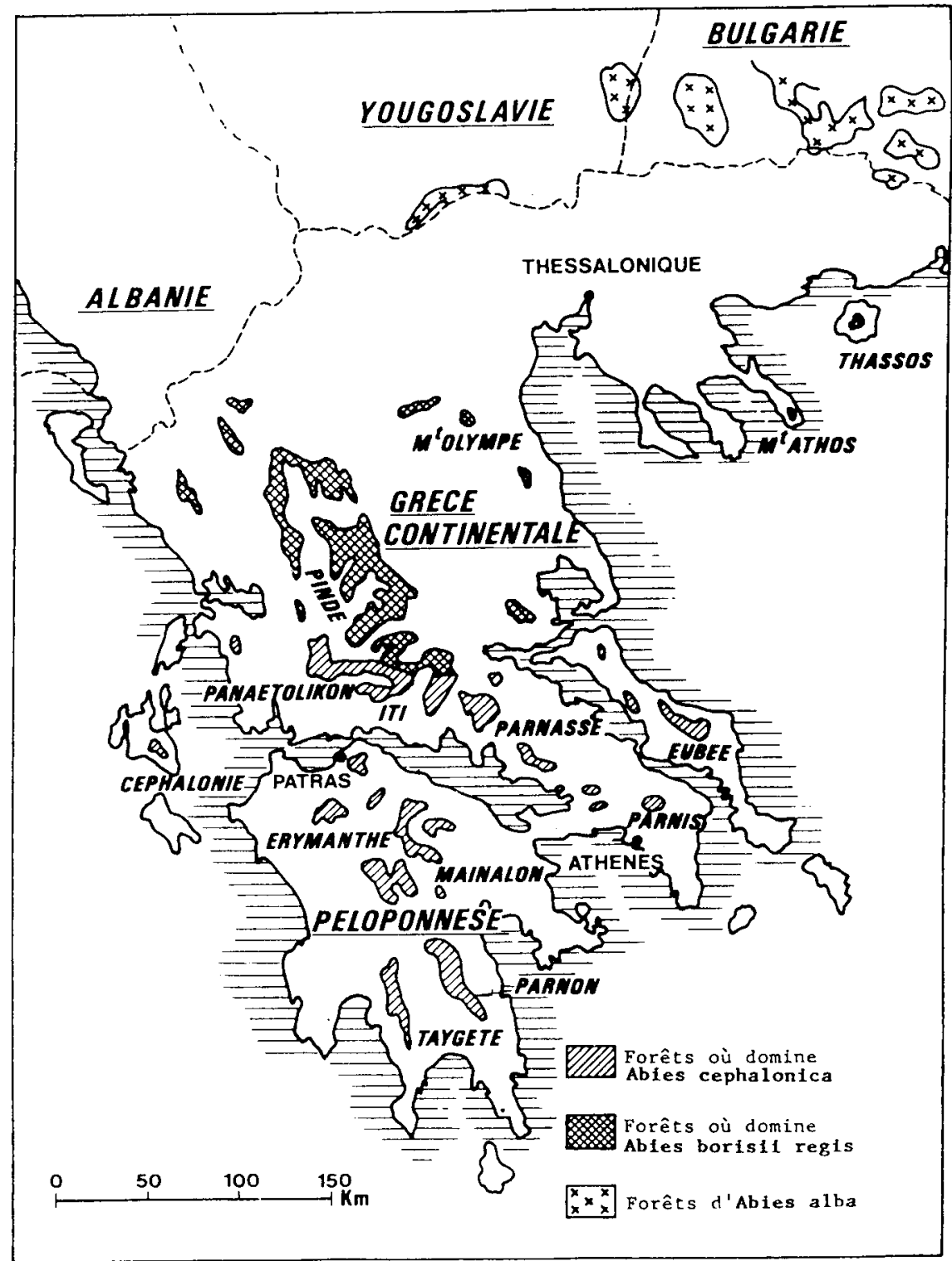

Fig 1. Aire de répartition des principales formations de sapins en Grèce.

1982 pour Pseudotsuga menziesii; Cannell et Smith, 1983 pour Picea sitchensis). En effet, il semble qu'un végétal ait surtout besoin, en liaison avec une variation de photopériode (Campbell et Sugano, 1975), d'une accumulation d'uni- tés de chaleur pour débourrer (en particulier dans le sol) après la levée de la dormance hivernale par les basses températures (Lavender, 1980). La date du $1^{\text {er }}$ janvier a été choisie arbitrairement comme le moment où le besoin du 
Tableau III. Description de l'échantillonnage utilisé dans l'étude de la précocité du débourrement.

\begin{tabular}{lcccccccccc}
\hline & Ceph & Erym & Veti & Pnon & Evia & Parn & Pnas & Oete & Pnae \\
\hline $\begin{array}{l}\text { Nombre d'individus } \\
\text { observés }\end{array}$ & 100 & 306 & 450 & 155 & 69 & 100 & 100 & 100 & 100 \\
$\begin{array}{l}\text { Nombre de familles } \\
\text { observées }\end{array}$ & - & 18 & 26 & 10 & 17 & - & - & - & - \\
$\begin{array}{l}\text { Nombre moyen } \\
\text { d'individus par famille }\end{array}$ & - & 17,0 & 17,3 & 15,5 & 4,0 & - & - & - & - \\
\hline
\end{tabular}

végétal en températures froides est remplacé par un besoin en unités de chaleur.

Le modèle général utilisé dans l'analyse statistique est du type :

$$
x_{i j k}=\mu+p_{i}+f_{i j}+r_{i j k}
$$

où $x_{i j k}$ est le caractère analysé (note de débourrement), $\mu$ la moyenne générale, $p_{i}$ l'effet de la provenance $i, f_{i j}$ l'effet de la famille $j$ dans la provenance $i$ et $r_{i j k}$ l'effet de l'individu $k$ dans la famille $j$ et la provenance $i$.

Les moyennes familiales ont été ajustées à l'effet bloc. Les moyennes de familles et de provenances ont été comparées par une analyse de variance non orthogonale hiérarchique à 2 niveaux et classées par le test de comparaison multiple de moyennes de Duncan.

Les coefficients de prédiction génétique (Baradat, 1976) ont été calculés sur l'ensemble des individus en supposant l'homogénéité des matrices de variance/covariance individuelles et familiales pour les différentes provenances et en utilisant la relation :

$$
\sigma^{2} x=\sigma^{2} p+\sigma^{2} f+\sigma^{2} r
$$

où $\sigma^{2} x$ représente la variance phénotypique du caractère $x(p$, provenance; $f$, famille; $r$, individu dans famille). Les valeurs de l'héritabilité au sens strict $\left(h^{2}\right)$ ont été calculées selon le modèle génétique exposé dans Falconer (1974), mais en tenant compte d'une consanguinité probablement importante en forêt naturelle. Une limite supérieure du taux de consanguinité $(F)$ a pu être évaluée à 0,6 à partir des fréquences des individus riches ou pauvres en un terpène, le limonène, dans un peuplement naturel d'Abies alba (Moreau, 1989). Une appréciation indirecte de cette valeur pour le même terpène donne une valeur de 0,7 pour Abies cephalonica (Fady, 1990). Shea (1990) montre dans une étude isoenzymatique portant sur un grand nombre de loci que le coefficient moyen de consanguinité chez Abies lasiocarpa est de 0,341 . Ainsi, en utilisant comme extrêmes des estimations moyennes de $F$ variant entre 0,3 et 0,6 :

$$
h^{2}=\frac{4}{1+F} \quad \frac{\sigma^{2} f}{\sigma^{2} f+\sigma^{2} r}
$$

d'où $2,5 \frac{\sigma^{2} f}{\sigma^{2} f+\sigma^{2} r} \quad<h^{2}<3,1 \quad \frac{\sigma^{2} f}{\sigma^{2} f+\sigma^{2} r}$

au niveau intra-provenance, en faisant une évaluation moyenne sur l'ensemble des provenances et

$$
\frac{2,5 \sigma^{2} f+\sigma^{2} p}{\sigma^{2} f+\sigma^{2} r+\sigma^{2} p}<h^{2}<\frac{3,1 \sigma^{2} f+\sigma^{2} p}{\sigma^{2} f+\sigma^{2} r+\sigma^{2} p}
$$

si la variance entre provenances est considérée comme une composante de la variance génétique : le calcul est alors réalisé toutes provenances confondues et la variance interprovenances s'ajoute à la variance interfamiliale intra-provenance.

\section{RÉSULTATS}

L'ajustement à l'effet bloc s'est révélé superflu, les tests $F$ de l'effet bloc étant non 
significatifs. Les microvariations climatiques et pédologiques n'ont pas d'influence significative sur la précocité du débourrement. Lacaze (1969) observait de même un effet bloc négligeable sur le débourrement de jeunes épicéas en plantation comparative.

De manière générale, les bourgeons terminaux de l'axe principal s'ouvrent plus tard (entre 2 et 4 semaines) que ceux des rameaux latéraux et le bourgeon terminal débourre plus tard (entre 1 et 2 semaines) que les bourgeons latéraux du dernier verticille de l'axe principal. Ce schéma de développement est comparable à celui des autres espèces de sapins (Franklin et Ritchie, 1970) et de Conifères en général (White et al, 1979; Lavender, 1980; Worrall, 1983).

En 1987, l'ensemble des provenances débourrent entre le 5 et le 11 mai (fig 2). Les 3 méthodes utilisées donnent des résultats rigoureusement concordants.

\section{Variabilité intra-provenance}

La variabilité entre familles d'une même provenance a été représentée sur la figure 3. L'amplitude de cette variabilité est semblable pour les provenances Erym, Veti et Pnon. Celle de la provenance Evia est apparemment beaucoup plus forte. Elle est en fait augmentée par l'existence d'une famille très précoce, ce qui se retrouve dans les coefficients de variation familiaux (tableau IV). II faut noter qu'une de ces familles (612) est tout à fait remarquable par le caractère tardif de son débourrement et sa forte croissance en hauteur. Le coefficient de variation individuel intrafamilial moyen est 2-3 fois plus important que le précédent.

La part de variation due à l'effet provenance est faible, puisqu'elle ne correspond qu'à $10 \%$ de la variabilité génétique totale.
Tableau IV. Coefficients de variation de l'indice de précocité de débourrement, calculés à partir de la méthode 1-2.

Erym Veti Pnon Evia Moyenne

$\begin{array}{llllll}\begin{array}{l}\text { Coefficient } \\ \text { interfamilial }\end{array} & 0,05 & 0,05 & 0,05 & 0,08 & 0,05 \\ \begin{array}{l}\text { Coefficient } \\ \text { intrafamilial }\end{array} & 0,15 & 0,15 & 0,15 & 0,13 & 0,15\end{array}$

Toutes ces remarques concordent et tendent à montrer que la variabilité individuelle (ie, en partie environnementale) est très importante et que la variabilité génétique est plus affirmée entre les familles d'une même provenance qu'entre différentes provenances.

Les héritabilités ont pu être calculées avec un niveau de signification suffisant pour 2 provenances (tableau $\mathrm{V}$ ) et sont tout fait comparables avec les héritabilités obtenues en regroupant l'ensemble des provenances : $h^{2}=0,19$ à 0,27 pour la méthode $1-2$ et $h^{2}=0,21$ à 0,31 pour la méthode 1-5. La faible augmentation de $h^{2}$ lorsque la composante provenance entre en compte dans l'estimation de la variance génétique indique encore que l'essentiel de la variation génétique se trouve entre les familles.

Les coefficients de corrélation entre hauteur totale et précocité du débourrement ne sont pas significatifs au niveau individuel intra-provenances, au niveau génétique et au niveau phénotypique.

\section{Variabilité interprovenance}

Les valeurs moyennes de l'indice de précocité de débourrement provenance par provenance permettent une bonne discrimination. Tous les tests $F$ sont significatifs 
La date correspond au moment où $50 \%$ des individus ont débourré

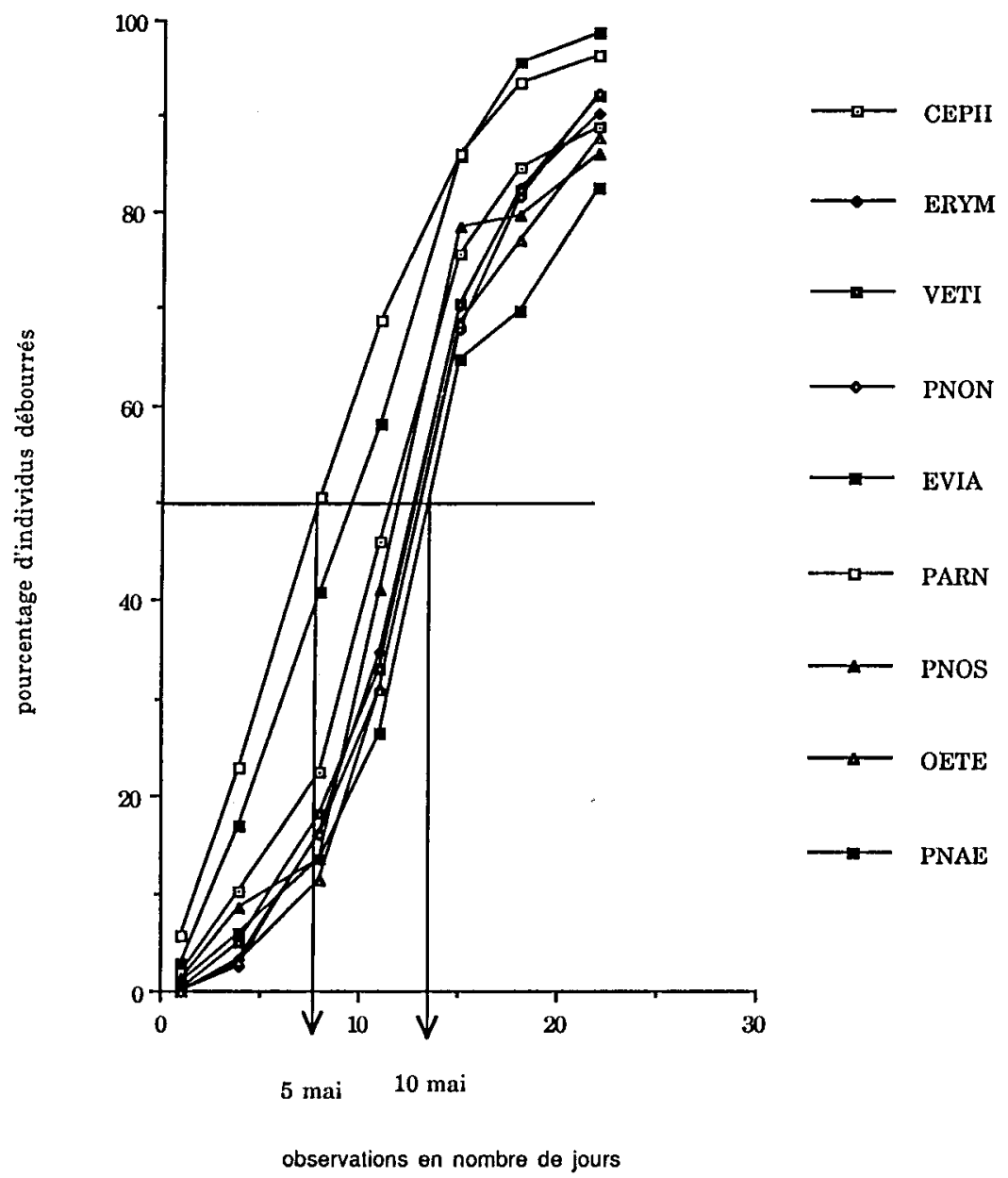

Fig 2. Dates de débourrement des provenances de St-Lambert en 1987.

au seuil de $1 \%$. Les tests de Duncan au seuil de $5 \%$ montrent que les provenances Parn et Evia sont les plus précoces. Les autres forment un ensemble où Ceph et Pnas sont relativement plus précoces et Pnae relativement plus tardive (fig 4).

Ce caractère est fortement corrélé $(r=$ $\left.-0,87^{\star \star}\right)$ avec la hauteur totale : les prove- nances les plus précoces ont aussi les hauteurs totales les plus faibles.

Une série de régressions simples de la précocité du débourrement sur les conditions climatiques du lieu d'origine de chaque provenance montre des corrélations hautement significatives avec l'aridité (indice de De Martonne, $\left.r=-0,80^{\star \star}\right)$, les pré- 


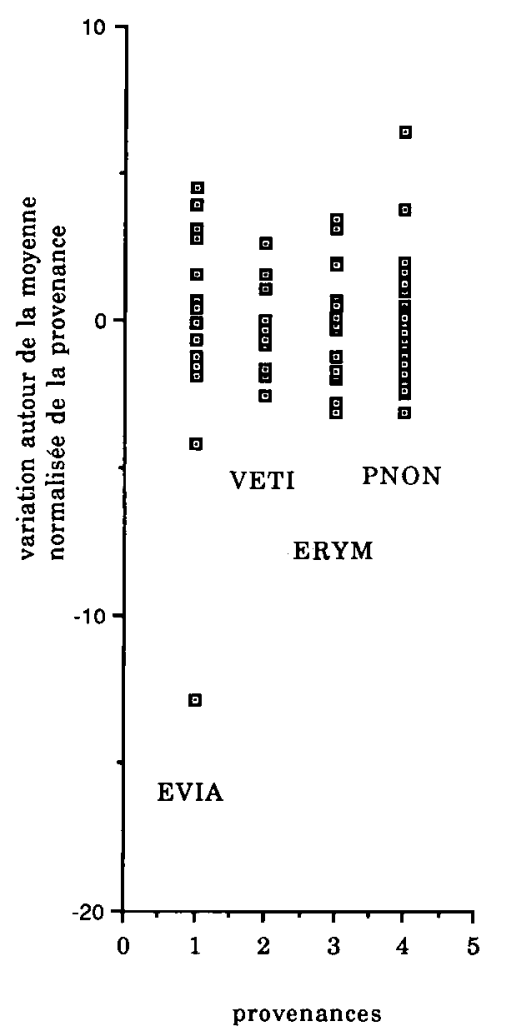

Fig 3. Allure de la variabilité intra-provenance du caractère débourrement.

cipitations annuelles $\left(r=-0,83^{* *}\right)$ et la continentalité $\left(r=-0,67^{\star}\right)$. Lorsque l'indice d'aridité du lieu augmente et que les précipitations annuelles et l'éloignement de la mer décroissent, les populations ont des débourrements plus précoces.

\section{Comparaison des 3 méthodes d'étude}

La corrélation entre les méthodes 1-2 et 15 , sur la plantation $V G_{1}$, est très satisfaisante $\left(r=0,90^{* \star}\right)$ aux niveaux provenance,

* Valeur significative au seuil de 5\%.

** valeur significative au seuil de $1 \%$. note de provenance
débourrement

\begin{tabular}{l|l}
11,21 & \\
10,97 & \\
10,32 \\
10,09 \\
10,01 \\
9,97 \\
9,93 \\
9,89 \\
9,60
\end{tabular}

Fig 4. Comparaison de la précocité du débourrement des provenances de Sapin de Céphalonie sur la plantation de St-Lambert en 1987, sur la base de la méthode d'observation débourré/ non débourré. Test de Duncan au seuil de $5 \%$ (toute provenance non reliée à une autre par une barre verticale lui est significativement différente pour la précocité de son débourrement).

famille ou individu. Cependant, la méthode 1-5 permet une discrimination plus fine des provenances puisque au seuil de $5 \%$, les provenances Ceph et Evia forment deux groupes distincts du reste des provenances, alors que pour la méthode 1-2, seule Evia se distingue des autres (fig 5).

L'utilisation de la méthode des sommes de températures n'apporte aucune amélioration par rapport à la méthode 1-2 pour la mise en évidence d'une variabilité génétique entre provenances.

\section{DISCUSSION}

Bien que la part de la variabilité génétique attribuable à l'effet provenance soit faible, plusieurs groupes de provenances apparaissent nettement. Parn, Evia et Ceph sont plus précoces que l'ensemble des autres provenances. Fady (1990) avait pu 
Tableau V. Valeurs de l'héritabilité calculées à partir de la méthode en 2 points (méthode 1-2) et de la méthode en 5 points (méthode 1-5). Les valeurs varient entre deux extrêmes correspondant aux limites inférieures et supérieures du coefficient moyen de consanguinité $(F) .{ }^{* *}$ : valeur significative au seuil de $1 \%$; NS : valeur non significative.

\begin{tabular}{|c|c|c|c|c|}
\hline \multirow{2}{*}{ Provenances } & \multicolumn{2}{|c|}{ Méthode 1-2 } & \multicolumn{2}{|c|}{ Méthode 1-5 } \\
\hline & $F=0,6$ & $F=0,3$ & $F=0,6$ & $F=0,3$ \\
\hline Erym & $0,19^{\star *}$ & $0,24^{\star \star}$ & $0,21^{\star *}$ & $0,26^{\star \star}$ \\
\hline Veti & $0,22^{* *}$ & $0,27^{\star *}$ & $0,25^{\star *}$ & $0,31^{* *}$ \\
\hline Pnon & NS & NS & NS & NS \\
\hline Evia & NS & NS & NS & NS \\
\hline $\begin{array}{l}\text { Moyenne avec la } \\
\text { composante provenance } \\
\text { Moyenne sans la }\end{array}$ & $0,21^{\star *}$ & $0,26^{* *}$ & $0,24^{\star \star}$ & $0,29^{* *}$ \\
\hline composante provenance & $0,20^{\star \star}$ & $0,25^{\star \star}$ & $0,23^{\star \star}$ & $0,29^{\star *}$ \\
\hline
\end{tabular}

METHODE 1-2

note de débourrement provenances

$\begin{array}{cc}10,96 & \text { EVIA } \\ 10,32 & \text { CEPH } \\ 9,99 & \text { VETI } \\ 9,95 & \text { ERYM } \\ 9,89 & \text { PNON }\end{array}$

METHODE 1-5

$\begin{array}{cc}\begin{array}{c}\text { note de } \\ \text { débourrement }\end{array} & \text { provenances } \\ 25,85 & \text { EVIA } \\ 23,79 & \text { CEPH } \\ 22,47 & \text { VETI } \\ 22,32 & \text { ERYM } \\ 22,16 & \text { PNON }\end{array}$

Fig 5. Comparaison des méthodes d’observation «débourré/non débourré» (méthode 1-2) et en «5 points" (méthode 1-5) sur le site VG1 à St-Lambert. Classement des provenances par le test de Duncan au seuil de $5 \%$ (toute provenance non reliée à une autre par une barre verticale lui est significativement différente pour la précocité de son débourrement.

mettre en évidence par l'étude de la croissance en hauteur et de la composition en monoterpènes et sesquiterpènes une séparation génétique analogue entre ces provenances, notamment entre le groupe "oriental» Evia-Parn et les populations du Péloponnèse et de Grèce continentale.

La méthode en 5 points est visiblement meilleure pour discriminer les provenances, et apporte une précision un peu supérieure dans le calcul des paramètres génétiques. La méthode des sommes de températures ne se justifiait sans doute pas dans la mise en évidence d'une plus grande variabilité du fait de la relative proximité de comportement des différentes provenances cette année-là. II serait intéressant de tester, en environnement contrôlé, l'importance de l'influence du facteur température sur la phénologie du 
bourgeon végétatif par rapport à d'autres facteurs, comme la photopériode par exemple (Niendstaedt, 1967 pour Picea sp; Eriksson et al, 1978 pour Picea abies).

L'absence de corrélation aux niveaux individuel et familial entre hauteur totale et précocité du débourrement montre qu'il n'y a pas de relation entre la précocité du débourrement du bourgeon terminal et sa distance du sol. II était en effet possible de craindre l'introduction d'un biais expérimental du fait, d'une part de l'existence d'un gradient de températures atmosphériques du plus chaud vers le plus froid au fur et à mesure que la proximité du sol augmente et, d'autre part, de l'amplitude des hauteurs totales individuelles recensées sur la plantation.

Par contre, une forte corrélation positive apparaît au niveau provenance entre vigueur et retard du débourrement. Une telle corrélation avait pu être mise en évidence notamment par Lacaze (1969) chez Picea abies, par Arbez et Millier (1970) chez Abies alba et par Cristophe et Birot (1979) chez Pseudotsuga menziesii. En France méditerranéenne, la sécheresse estivale n'est pas aussi accusée qu'en Grèce, par contre les risques de gelées tardives $y$ sont beaucoup plus importants (Plantation INRA de Pélenq dans le Haut-Var, par exemple, où les bourgeons latéraux de 3 années de végétation ont été détruits par le gel, conduisant à un aspect général columnaire de l'arbre). Ainsi, la sélection de provenances vigoureuses et tardives est tout à fait intéressante en vue de reboisements.

La variabilité du débourrement peut être interprétée comme le reflet d'une sélection naturelle. Les provenances ont pu s'adapter différemment selon les conditions écologiques de leur lieu d'origine, de manière à "optimiser leur date de débourrement" (Campbell et Sugano, 1979). Ces conditions écologiques agissent de manière va- riable sur la phénologie. Les observations de certains auteurs (Kozlowski, 1964; White et al, 1979; Lavender, 1980) tendent à montrer que les provenances ou écotypes originaires des régions les plus chaudes de l'aire naturelle ont les dates de débourrement les plus précoces, probablement en relation avec un besoin en basses températures plus court pendant la période de dormance (Nienstaedt, 1967 pour Picea $\mathrm{sp})$. Pour White et al (1979), la précocité du débourrement de Pseudotsuga menziesii est due en particulier au manque de précipitations estivales. D'autres auteurs montrent en revanche que ce sont les provenances situées aux plus hautes altitudes et latitudes qui débourrent les premières (Steiner, 1979; Worrall, 1983).

Dans le cas du sapin de Céphalonie, il semblerait que la chaleur ait un rôle indirect et que l'aridité, par le jeu de l'éloignement de la mer et du manque de précipitations, soit le principal responsable de cette adaptation. Steiner (1979) mentionne pour plusieurs espèces de pins européens le rôle localement important de la proximité de la mer. Ces facteurs climatiques locaux peuvent expliquer l'absence de gradient géographique nord-sud de débourrement, tel qu'il avait été observé pour la morphologie (Fady et al, 1990).

Deux types de stratégies adaptatives semblent devoir s'opposer lorsque la saison de végétation est limitée, l'une vis-àvis du froid, l'autre vis-à-vis de la sécheresse estivale, selon la situation écologique de l'espèce (Campbell et Sugano, 1979). Le débourrement précoce des provenances "xérophiles" de sapin de Céphalonie et le débourrement précoce de ce sapin par rapport aux autres sapins méditerranéens (Fady et al, 1990) peut sans doute être interprété comme le reflet d'une sélection entre les risques (moindres dans ce cas) que représentent les gelées tardives et ceux que représente le stress hy- 
drique estival. Ainsi, aucun dégât de gel n'a pu être observé dans l'aire naturelle (observations personnelles, 1988).

La structure de la variabilité semble sensiblement la même pour toutes les provenances. La valeur de l'héritabilité est très constante d'une provenance à l'autre et semble donc pouvoir être extrapolée à l'ensemble de l'espèce. Cette valeur est comprise entre 0,19 et 0,31 . A titre comparatif, la valeur de l'héritabilité de ce caractère variait entre 0,28 et 0,51 pour de jeunes épicéas, en faisant abstraction d'éventuels effets de consanguinité (Lacaze et Arbez, 1971). Cette valeur d'héritabilité reste modérée pour un caractère stable et déterminé génétiquement comme le suppose Lacaze (1970) pour Picea abies, probablement à cause d'une variance environnementale élevée (Thielges et Beck, 1976). Nienstaedt (1972) note que l'héritabilité du débourrement de Picea glauca calculée en milieu contrôlé (serre), est nettement supérieure à celle qui est obtenue en plantation. Sur la plantation de Saint-Lambert, les températures du début mai 1987 sont devenues brutalement très élevées, après un hiver relativement froid. Le seuil d'unités de chaleur a dû être atteint très rapidement et de manière uniforme pour toutes les provenances, conduisant à un débourrement littéralement explosif. Ainsi, les différences entre familles auraient été réduites devant l'effet environnement, ce que tendraient à prouver les coefficients de variations intrafamiliaux 2-3 fois plus élevés que les coefficients de variation entre familles. White et al (1979) ont montré chez Pseudotsuga menziesii, les années où le débourrement était plus précoce, que les provenances avaient tendance à se regrouper et ainsi effacer artificiellement leurs différences.

Les valeurs de l'héritabilité sont cependant suffisamment élevées pour une sélection efficace de provenances et de fa- milles à la fois vigoureuses et tardives, capables d'éviter les dernières gelées de printemps. C'est généralement le cas des provenances du Péloponnèse Pnon, Veti et Erym, mais certaines familles de la provenance Evia sont tout à fait remarquables de ce point de vue.

\section{RÉFÉRENCES}

Arbez M (1969) Étude comparative en pépinière de quelques provenances françaises de Sapin pectiné (Abies alba Mill); premier aperçu de la variabilité infraspécifique et mise au point sur le sapin de l'Aude. Ann Sci For 26, 475-509

Arbez M, Millier C (1970) Comparaison de deux générations successives de Sapin (Abies alba Mill). Structure génétique de population, hypothèses sur l'action de la sélection naturelle. Ann Sci For 27, 287-301

Baradat P (1976) Use of juvenile-mature relationships and information from relatives in combined multitrait selection. Proc IUFRO joint meet Advanced Generation Breeding, Bordeaux, France, 121-138

Barbero M, Quézel P (1976) Les groupements forestiers de Grèce Centro-Méridionale. Ecol Mediterr 2, 3-86

Birot Y, Ferrandes P (1972) Quelques aspects de la variabilité infraspécifique du Douglas (Pseudotsuga menziesii Mirb) introduit en zone méditerranéenne subhumide. Ann Sci For 29, 335-351

Bornovas J, Rondogianni-Tsiambaou T (1983) Geological map of Greece (1/500 000). Athens, 2nd ed

Campbell RK, Sugano Al (1975) Phenology of bud-burst in Douglas-fir related to provenance, photoperiod, chilling and flushing temperaturé. Bot Gaz 136, 290-298

Campbell RK, Sugano Al (1979) Genecology of bud-burst phenology in Douglas-fir: response to flushing temperature and chilling. Bot Gaz 140, 223-231

Cannell MGR, Smith RI (1983) Thermal time, chill days and prediction of budburst in Picea sitchensis. J Appl Ecol 20, 951-963 
Cristophe C, Birot $Y$ (1979) Genetic variation within and between populations of Douglasfir. Silvae Genet 28(5-6), 197-206

Debazac EF (1965) Observations sur le débourrement et la croissance en longueur de quelques espèces de Sapins. Rev For Fr 2, 120130

Debazac EF (1967) Nouvelles observations sur le débourrement et la croissance en longueur de quelques especes de Sapins. Rev For Fr 3, 183-190

Eriksson G, Ekberg I, Dormling I, Matern B, Von Wettstein D (1978) Inheritance of bud-set and bud-flushing in Picea abies (L) Karst. Theor Appl Genet 52, 3-19

Fady B (1990) Variabilité génétique du Sapin de Grèce. Thèse de doctorat, université AixMarseille 3

Fady B, Arbez M, Ferrandes P (1990) Variability of juvenile Greek Firs (Abies cephalonica Loud) and stability of characteristics with age. Silvae Genet (in press)

Falconer DS (1974) Introduction à la génétique quantitative. Masson, Paris

Franklin JF, Ritchie GA (1970) Phenology of cone and shoot development of noble Fir and some associated true firs. For Sci 16, 356364

Kozlowski TT (1964) Shoot growth in woody plants. Bot Rev 30(3), 335-392

Lacaze JF (1969) Étude de la variabilité infraspécifique de l'épicéa (Picea abies Karst). Provenances françaises et polonaises. Résultats au stade juvénile. Ann Sci For 26, 345-396

Lacaze JF (1970) Analyse d'une expérience multi-stationnelle de provenances d'épicéa (Picea abies Karst). Ann Sci For 27, 5-37

Lacaze JF, Arbez M (1971) Variabilité infraspécifique de l'épicéa (Picea abies Karst, partie septentrionale de l'aire française). Héritabilité et corrélations génétiques de quelques caractères au stade juvénile. Ann Sci For 28, $141-183$

Lacaze JF, Tomassone R (1967) Contribution à l'étude de la variabilité infraspécifique d'Abies grandis Lindl. Caractéristiques juvéniles. Ann Sci For 24, 277-325

Lavender DP (1980) Effects of the environment upon the shoot growth of woody plants. In:
Control of shoot growth in trees, proc IUFRO meet Fredericton, New Brunswick, Canada, July 1980, 76-106

Mauget JC (1983) Étude de la levée de dormance et du débourrement des bourgeons de Noyer (Juglans regia L, CV "Franquette") soumis à des températures supérieures à $15^{\circ} \mathrm{C}$ au cours de leur période de repos apparent. Agronomie 3, 745-750

Mauget JC (1987) Dormance des bourgeons chez les arbres fruitiers de climat tempéré. In: Le développement des végétaux, aspects théoriques et synthétiques ( $\mathrm{H}$ Le Guyader ed), Masson, Paris, 133-150

Moreau MC (1989) Contribution à l'étude de la structure génétique d'un peuplement de sapin pectiné (Abies alba Mill) des Basses Vosges. DEA d'Écologie expérimentale, université de Pau et des pays de l'Adour.

Nienstaedt $H$ (1967) Chilling requirements in seven Picea species. Silvae Genet 16(2), 6568

Nienstaedt H (1972) Degree-day requirements for bud flushing in white Spruce: variation and inheritance. Proc 8th Central States For Tree Improvement Conf, Columbia MO, Oct 11-15 1972, 28-32

Shea KL (1990) Genetic variation between and within populations of Engelmann spruce and subalpine fir. Genome 33, 1-8

Steiner KC (1979) Patterns of variation in budburst timing among populations in several Pinus species. Silvae Genet 28, 185-194

Thielges BA, Beck RC (1976) Control of budbreak and its inheritance in Populus deltoides. In: Tree physiology and yield improvement (MGR Cannell and FT Last eds), Acad Press, London, 253-260

Thompson AJ, Moncrieff SM (1982) Prediction of bud burst in Douglas-fir by degree-day accumulation. Can J For Res 12, 448-452

White TL, Ching KK, Walters J (1979) Effects of provenance, years, and planting location on bud burst of Douglas-fir. For Sci 25, 161-167

Worrall J (1983) Temperature - budburst relationship in amabilis and subalpine Fir. Provenance tests replicated at different elevations. Silvae Genet 32, 203-209 